\title{
Isolation of notoamide E, a key precursor in the biosynthesis of prenylated indole alkaloids in a marine-derived fungus,
}

\section{Aspergillus sp}

\author{
Sachiko Tsukamoto ${ }^{\star}, \dagger, \ddagger$, Hikaru Kato ${ }^{\dagger}$, Thomas J. Greshock $§$, Hiroshi Hirota ${ }^{\perp}$, Tomihisa \\ Ohta $†$, and Robert M. Williams ${ }^{\star}, \S, \|$ \\ Graduate School of Science, Chiba University, 1-33 Yayoi-cho, Inage-ku, Chiba 263-8522, Japan, \\ Graduate School of Natural Science and Technology, Kanazawa University, Kanazawa 920-1192, \\ Japan, Department of Chemistry, Colorado State University, Fort Collins, CO 80523 USA, RIKEN \\ Genomic Sciences Center (GSC), 1-7-22 Suehiro-cho, Tsurumi-ku, Yokohama 230-0045, Japan, \\ and the University of Colorado, Cancer Center, Aurora, Colorado 80045, USA
}

\begin{abstract}
Microorganisms are known to be rich sources of biologically active natural products that contain unique structures. The fungi of the genera Aspergillus and Penicillium are known to produce prenylated indole alkaloids, which are derived from tryptophan, proline, and one or two isoprene units constituted of diverse ring systems. Among them, the brevianamides, 1 paraherquamides, ${ }^{2}$ stephacidins, ${ }^{3}$ and avrainvillamide ${ }^{4}$ possess a characteristic bicyclo[2.2.2] diazaoctane ring, and have become enticing targets for synthetic studies. Recently, we reported the structurally related metabolites, notoamides A-D (1-4), 5 isolated from a marine-derived fungus, identified as an Aspergillus sp. along with the known alkaloids sclerotiamide, ${ }^{6}$ deoxybrevianamide E and stephacidin A (5) (Figure 1). It is noteworthy that the marine-derived Aspergillus sp., which we have investigated, exhibits an extensive co-metabolite profile representative of the structurally diverse ring systems of this family of prenylated indole alkaloids. The metabolite profile of this organism hinted at a possible biosynthetic pathway for these alkaloids, and we postulated the structure of a key, advanced intermediate $\mathbf{6}$ for the notoamides (herein named notoamide E) (Figure 1). ${ }^{7}$ Although we tried to isolate 6, it was not found in the extract of our Aspergillus sp., which was cultured for 20 days. We have since embarked on a detailed biosynthetic investigation of notoamide biosynthesis employing our marine-derived Aspergillus sp. by first interrogating this organism for the production of $\mathbf{6}$ and secondly through feeding experiments with synthetic, doubly ${ }^{13} \mathrm{C}$-labelled $\mathbf{6}$.
\end{abstract}

The absence of the putative precursor 6 in the 20-day-old culture indicated that 6 might be a short-lived precursor. We then attempted to detect the presence of $\mathbf{6}$ over shorter time courses by HPLC and NMR, deploying an authentic, synthetic reference sample. The fungus was cultured on agar medium, and the metabolite profile was analyzed daily by HPLC. To confirm the presence of $\mathbf{6}$, we examined the NMR spectra of fractions with retention times corresponding to that of the synthetic sample. The spectra (Figure 2) revealed that 6 existed only in the culture incubated for five days and disappeared the next day. These results clearly

E-mail: E-mail: sachiko@ faculty.chiba-u.jp (ST) and E-mail: rmw@ chem.colostate.edu (RMW).

† Chiba University

* Kanazawa University

$\S_{\text {Colorado State University }}$

$\perp_{\text {RIKEN GSC }}$

$\|_{\text {The University of Colorado }}$

Supporting Information Available: The detail of experimental procedure, structure determination of 8-10, and NMR data. This material is available free of charge via the Internet at http://pubs.acs.org. 
indicated that 6 was produced by the fungus in the early phase of growth and was presumably rapidly converted to other down-stream metabolites. To confirm the structure, we cultured the fungus on agar medium in 175 plates and succeeded in the isolation of $6(0.63 \mathrm{mg})$ and the assignment of the NMR data (Table S1).

Subsequently, we carried out precursor feeding and incorporation experiments with synthetic, doubly ${ }^{13}$ C-labelled 6.

First, the fungus was cultured in the normal nutrient-rich medium for a week. Then, the cells were successively cultured in trace element solution with the labeled precursor. After seventeen days, the culture was extracted with $n$ - $\mathrm{BuOH}$ and the extract containing the notoamides was purified with ODS HPLC to afford notoamides C (3) and D (4) and 3-epi-notoamide C (7)

$7 \mathrm{a}$ as well as three new metabolites that we have named notoamides E2-E4 (8-10) (Figure 1), which were all enriched with ${ }^{13} \mathrm{C}$ at the $\mathrm{C}-12$ and $\mathrm{C}-17$ positions. However, contrary to our expectation, compounds containing a bicyclo[2.2.2]diazaoctane ring such as notoamides A (1) and B (2) or stephacidin A (5) were not detected at all, although they are typically the major metabolites produced from this organism. This result strongly suggests that metabolites constituted of the bicyclo[2.2.2]diazaoctane ring system, including $\mathbf{1}, \mathbf{2}$ and $\mathbf{5}$, are not biosynthesized via $\mathbf{6}$ as originally proposed. ${ }^{7}$ The structures of the new alkaloids 8-10 were elucidated by analyses of their NMR and mass spectra (see Supporting Information). The structure of $\mathbf{1 0}$ is quite novel in the family of prenylated indole alkaloids, and two possible biosynthetic pathways to $\mathbf{1 0}$ via $\mathbf{6}$ are shown in Scheme 1. One of the possible pathways is via a peroxide species (14), which would arise by proton-assisted nucleophilic attack of the indole 3 -position on molecular oxygen to give 14. Another pathway would arise from $\mathbf{6}$ by singlet oxygen reaction at the indole 2,3-position to form dioxetane intermediate $\mathbf{1 1}$ followed by fragmentation to the corresponding kynurenine derivative 12.8 Then, cyclization of $\mathbf{1 2}$ would afford the eight-membered ring derivative $\mathbf{1 3}$ followed by dehydration would yield $\mathbf{1 0}$.

In conclusion, we have isolated notoamide $\mathrm{E}(\mathbf{6})$, which was previously postulated to be a putative biosynthetic precursor of the notoamides, from cultures of our marine-derived Aspergillus sp. Interestingly, $\mathbf{6}$ appeared only in the early phase of fungal growth and immediately disappeared. We have demonstrated through precursor feeding and incorporation experiments with ${ }^{13} \mathrm{C}$-labelled $\mathbf{6}$ in the minimal medium, that this substance was incorporated into notoamides $\mathrm{C}(\mathbf{3})$ and $\mathrm{D}(\mathbf{4})$ and 3-epi-notoamide $\mathrm{C}(7)$ along with three new minor alkaloids, notoamides E2-E4 (8-10). Although 7 was not isolated from the culture in the normal medium, the yield of $\mathbf{7}$ in the feeding experiment was more than that of $\mathbf{3}$.

Surprisingly, compounds containing a bridged bicyclo[2.2.2]diazaoctane core, such as notoamides A (1) and B (2) and stephacidin A (5), were not isolated from this feeding experiment. On the other hand, the incorporation of $\left[{ }^{3} \mathrm{H}\right]$ deoxybrevianamide $\mathrm{E}$ into brevianamides A and B was carried out in Penicillium brevicompactum, ${ }^{9}$ which clearly showed that the bicyclo[2.2.2] diazaoctane ring was constructed from the reverse prenyl group and the diketopiperazine ring in this organism. Recently, it was revealed that the closely related organism Aspergillus versicolor NRRL 35600 produces the opposite enantiomers of $\mathbf{2}$ and $\mathbf{5}$. 10 Therefore, the construction of the bicyclo[2.2.2] diazaoctane ring system in our marinederived Aspergillus sp. is an interesting enigma, and the search for the progenitor for $\mathbf{1 , 2}$ and $\mathbf{5}$ is under active investigation. The alkaloids 8-10 were not isolated from the culture of Aspergillus sp. in the normal nutrient rich medium. This result suggests that the presence of excess 6 in the growth medium alters the metabolite profile of this marine-derived fungus. Finally, notoamide E4 (10) constitutes a new type of alkaloid within this family, and the biogenesis of this structure is an interesting subject of current investigation. 


\section{Supplementary Material}

Refer to Web version on PubMed Central for supplementary material.

\section{Acknowledgements}

This work was supported by Grants-in-Aid for Scientific Research (No. 18032033 and 19310140) from the Ministry of Education, Culture, Sports, Science and Technology of Japan and also by grants from the Naito Foundation and the Keimeikai Foundation for Pharmaceutical Sciences. NIH support is also gratefully acknowledged (CA70375 to RMW).

\section{References}

1. (a) Birch AJ, Wright JJ. Tetrahedron 1970;26:2329-2344. [PubMed: 5419195] (b) Steyn PS. Tetrahedron Lett 1971:3331-3334. (c) Birch AJ, Russel RA. Tetrahedron 1972;28:2999-3008. (d) Steyn PS. Tetrahedron 1973;29:107-120.

2. (a) Yamazaki M, Okuyama E, Kobayashi M, Inoue H. Tetrahedron Lett 1981;22:135-136. (b) Ondeyka JG, Goegelman RT, Schaeffer JM, Kelemen L, Zitano L. J Antibiotics 1990;43:1375-1379. [PubMed: 2272914] (c) Liesch JM, Wichmann CF. J Antibiotics 1990;43:1380-1386. [PubMed: 2272915] (d) Blanchflower SE, Banks RM, Everet JR, Manger BR, Reading C. J Antibiotics 1991;44:492-497. [PubMed: 2061192] (e) Blanchflower SE, Banks RM, Everet JR, Reading C. J Antibiotics 1993;46:1355-1363. [PubMed: 8226314]

3. Qian-Cutrone J, Haung S, Shu Y-Z, Vyas D, Fairchild C, Menendez Z, Krampitz K, Dalterio R, Klohr SE, Gao Q. J Am Chem Soc 2002;124:14556-14557. [PubMed: 12465964]

4. a) Fenical, W.; Jensen, PR.; Cheng, XC. Avrainvillamide, a Cyttotoxic Marine Natural Product, and the Derivatives Thereof. 6,066,635. 2000. (b) Sugie Y, Hirai H, Inagaki T, Ishiguro M, Kim YJ, Kojima Y, Sakakibara T, Sakemi S, Sugiura A, Suzuki Y, Brennan L, Duignan J, Huang LH, Sutcliffe J, Kojima N. J Antibiotics 2001;54:911-916. [PubMed: 11827033]

5. Kato H, Yoshida T, Tokue T, Nojiri Y, Hirota H, Ohta T, Williams RM, Tsukamoto S. Angew Chem, Int Ed 2007;46:2254-2256.

6. Authrine C, Gloer JB. J Nat Prod 1996;59:1093-1095. [PubMed: 8946752]

7. (a) Grubbs AW, Artman GD III, Tsukamoto S, Williams RM. Angew Chem, Int Ed 2007;46:22572261. (b) Greshock TJ, Grubbs AW, Tsukamoto S, Williams RM. Angew Chem, Int Ed 2007;46:22622265.

8. Baran PS, Guerrero CA, Corey EJ. J Am Chem Soc 2003;125:5628-5629. [PubMed: 12733890]

9. Sanz-Cervera JF, Glinka T, Williams RM. Tetrahedron 1993;49:8471-8482.

10. Greshock TJ, Grubbs AW, Jiao P, Wicklow DT, Gloer JB, Williams RM. Angew Chem, Int Ed 2008;47:3573-3577. 


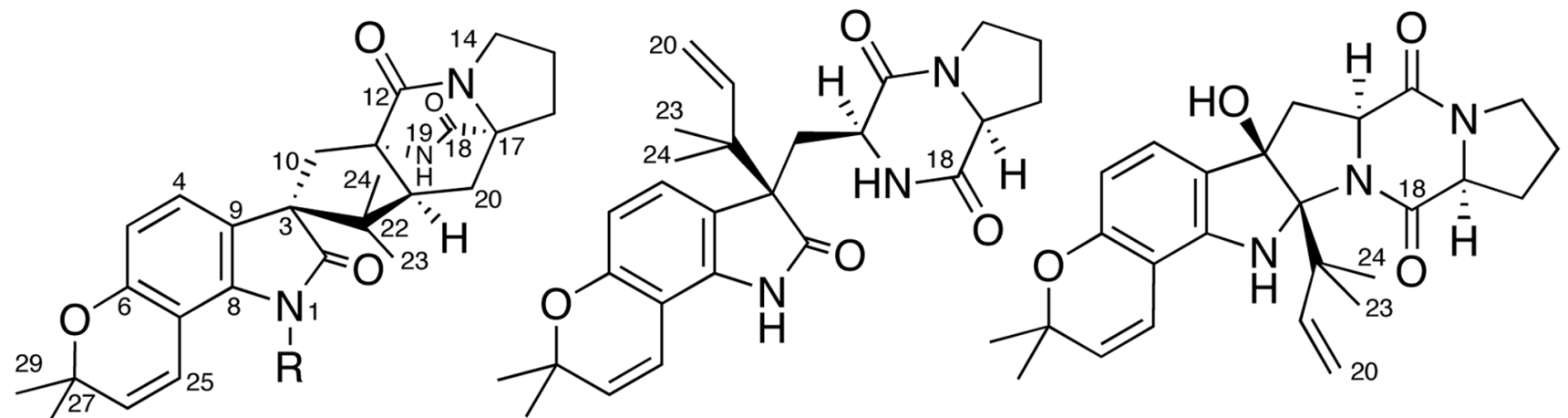

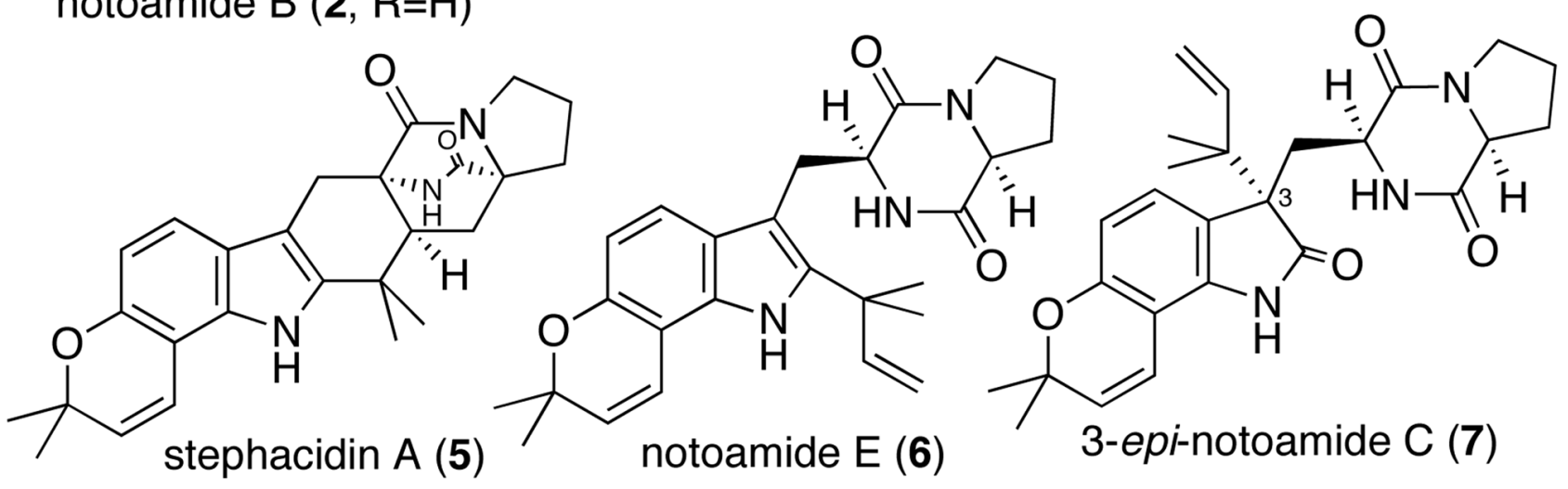<smiles>C=CC(C)(C)c1[nH]c2c3c(ccc2c1C[C@H]1NC(=O)[C@@H]2CCCN2C1=O)OC(C)(C)[C@H](O)[C@H]3O</smiles>

Figure 1.

Structures of compounds 1-6 isolated from the culture of Aspergillus sp. Compounds 7-10 were metabolites isolated only from the feeding experiment with ${ }^{13} \mathrm{C}$-labelled $\mathbf{6}$. In 8 and $\mathbf{9}$, the stereochemistry of the diol moieties has not yet been assigned relative to that of the dioxopiperazine moieties. 
(a)

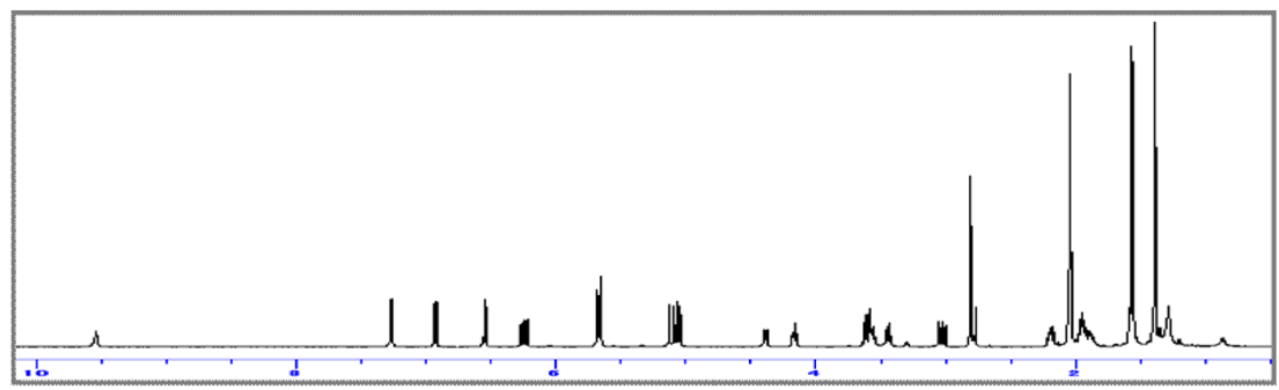

(b)

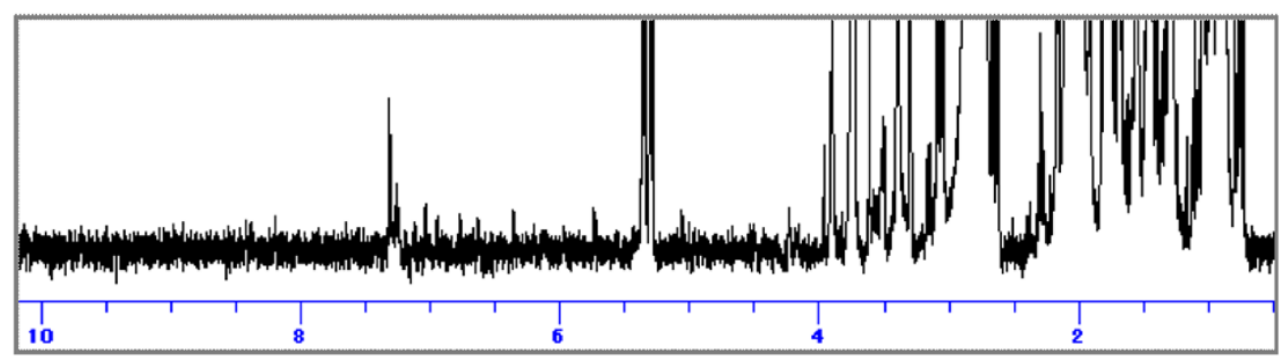

(c)

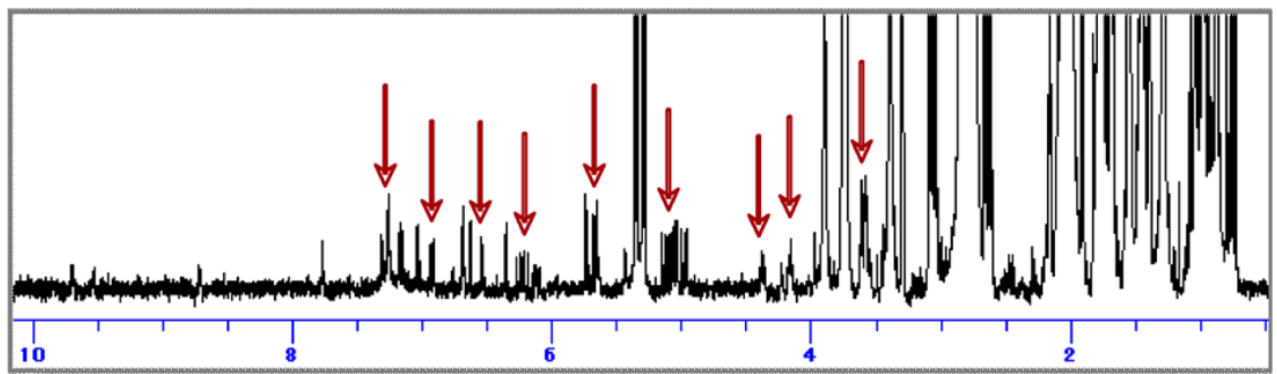

(d)

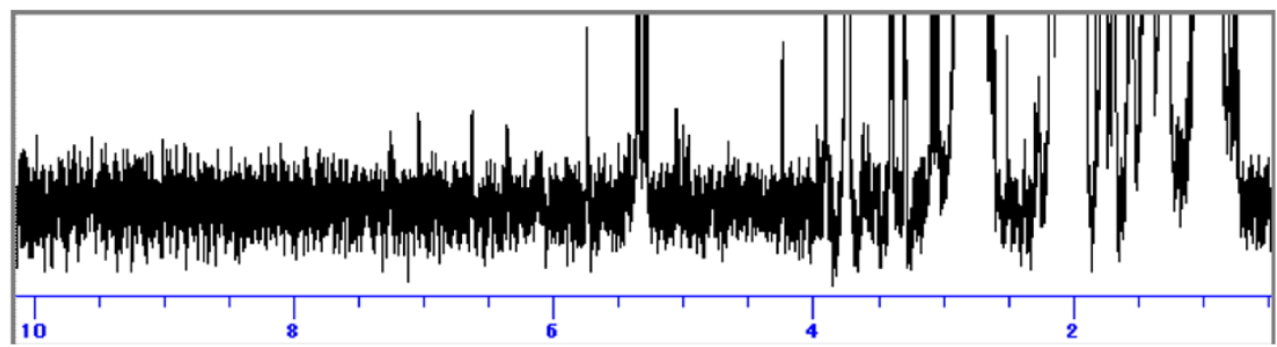

Figure 2.

NMR spectra (acetone- $d_{6}$ ) of fractions, of which retention time corresponded to 6 . Synthetic compound (a) and the fractions prepared from the culture incubated for four (b), five (c), and six (d) days. The red arrows show the signals for $\mathbf{6}$. 


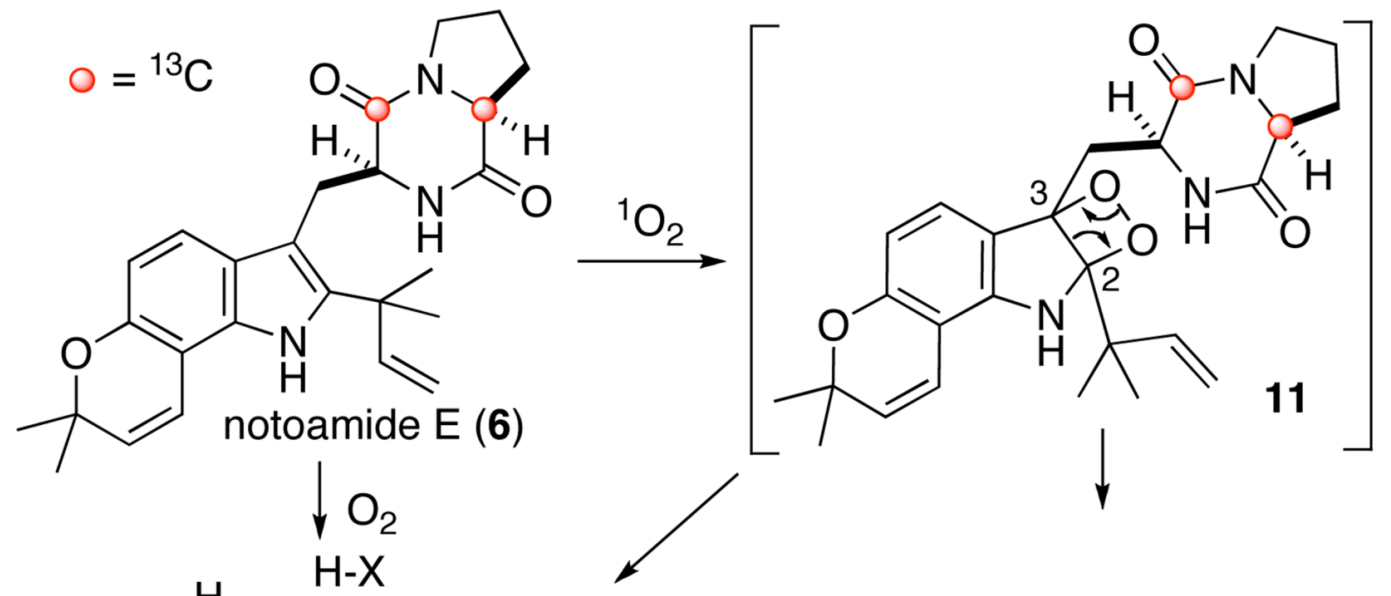

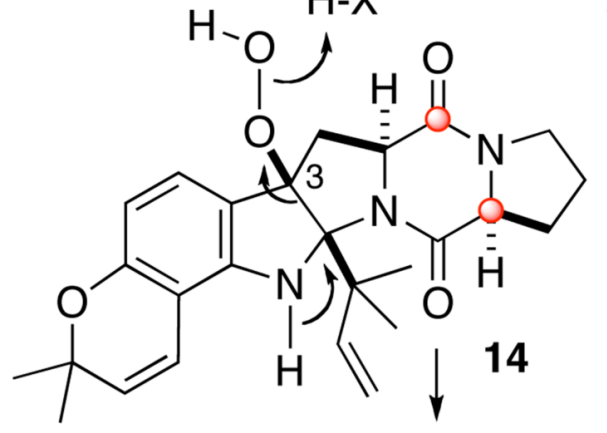

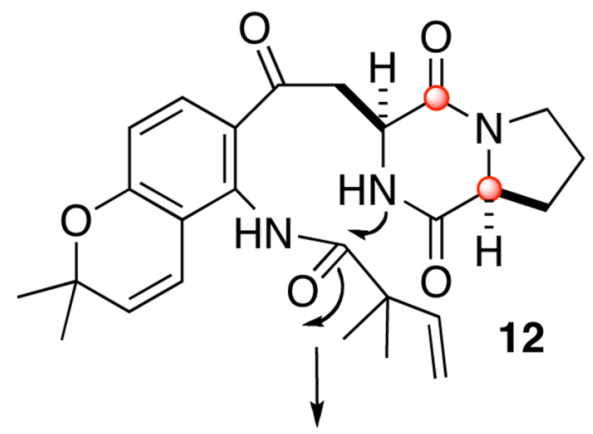

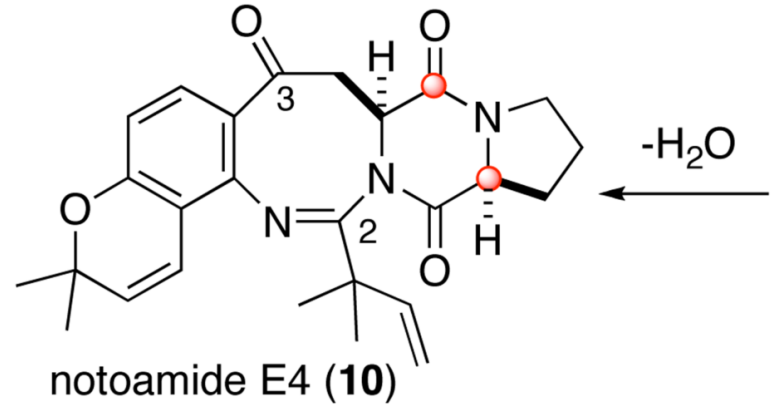<smiles>C=CC(C)(C)C1(O)Nc2c(ccc3c2C=CC(C)(C)O3)C(=O)C[C@@]2(C)C(=O)N3CCC[C@H]3C12C(=O)O</smiles>

Scheme 1.

Possible biosynthetic pathways to $\mathbf{1 0}$ via $\mathbf{6}$. 